\title{
Research on Maintenance Support System Based on Complex Network
}

Yang Ke ${ }^{1, a}$, Fan Shi-dong ${ }^{1}$ and Yao Yu-nan ${ }^{1}$

1 School of Energy and Power Engineering, Wuhan University of Technology, Wuhan, 430063, China.

\begin{abstract}
The design and collaboration analysis of maintenance support system network is a critical challenge. So the network model of ship maintenance support system was proposed, it involved all levels of distributed maintenance support participating nodes and formed a net chain structure. The network characteristic was analysed using complex network theory. The impact of topology characteristic and dynamic evolution on the cooperation effectiveness of maintenance support system was discussed. And an optimal evolution algorithm of support network was put up to so that the optimal support network would be designed.
\end{abstract}

\section{Introduction}

Maritime transport carries more than $80 \%$ of the international cargo traffic in the commodity exchange of economic globalization because of the advantages of capacity and cost. At the same time, the research on ship maintenance support based on operational reliability and economy has been widely concerned at home and abroad because of the competition in shipping market. More and more technology has been used to improve the reliability of state perception, which comes to a relatively accurate maintenance support prediction [1]. It is common for scholars to make a number of research on how to optimal the time window related to maintenance and support.

However, there has been a great gap from the maintenance demands to support response. Therefore the maintenance support supply chain network has become an effective means to improve maintenance benefits and operational collaboration.

Maintenance support system is driven by the maintenance requirements of ship equipment based on the supporting facilities. It controls at all levels of maintenance support resources dispersed in different locations through centralized or distributed information. Finally it forms an interrelated and closed collaboration system to achieve the supply, transport, allocation, collaboration and sharing of various types of resources[2]. It shows a high degree of networking in the geographical layout and operation relationship. Yin [3] abstracted the nodes of maintenance entities based on the flow and redistribution of elements in the maintenance implementation process. $\mathrm{He}$ established the topological meta-model and network relationship of maintenance support system. Through the change of statistical characteristic variables in the network structure, the influence of topology scale and information sharing and interaction ability on the efficiency of the support system is studied. Also the integrated mode of maintenance support is preliminarily discussed. Zhang [4] set up the perspective of complex network to study the command and control information synergy model. The synergy mechanism of uncertain supply and demand is studied from the view of case reasoning and Agent.

In general, the ship operation and maintenance supply chain is a complex network chain structure which revolves around the network nodes such as maintenance bases, suppliers, retailers, logistics enterprises and users. It mainly has the following characteristics:

1) Complexity. The operation and maintenance supply chain covers the entire process from raw material suppliers, parts suppliers, product manufacturers, retailers, service providers to terminal users. It is a large span structural pattern with multi-layer and multi-level participating entities, and enterprise nodes of different types, different geographies, different market positions, which all embody the complexity of maintenance supply chain network.

2 ) Dynamics. Firstly, node enterprises enter or leave the maintenance support network because of the random demand and the impact of the market environment. Secondly, due to the technological innovation and different business strategies of upstream and downstream node enterprises, the core

a Corresponding author: ake1231@163.com 
enterprises change the supply chain cooperation relationship dynamically.

3 ) Self-organization. The maintenance support supply chain is composed of independent node enterprises such as core maintenance bases, resource suppliers, manufacturers and end users. They make continuous exchange of raw materials, energy, capital, labor, technical services and information witl the outside world. There is competition and game between supply chain network node entities for individual interests. However, it finally comes to the co-operation on the basis of total network income by self-organization.

\section{Construction and evolution analysis of ship maintenance support network model}

The network research was first derived from the 20th century mathematician Euler's proof of the "Konigsberg seven Bridge Problem", which is typically characterized by the use of nodes and edges to abstract the operation of actual system. Nodes represent different individuals in the actual system, while edges represent a specific relationship between nodes (such as decision flow, information flow, logistics, capital flow, etc.)[5].

The onshore maintenance support entities involve the task ports, the supply stations, the repair shops, the shipyards, spare parts inventory at all levels and the suppliers in geographical dispersion. As a result, the network contains a series of sub-networks, such as organizational network, logistics supply network, inventory network, maintenance network, transportation network and so on.

As a result, the maintenance participating entities are divided into maintenance implementation layer and resource supply layer according to the attribute characteristics of the participating entities. Taking each maintenance support entity as the node, the maintenance support network model (shown in Fig.1) is abstracted from the geospatial layer on the edge of the interaction relationship (information flow, logistics, capital flow, etc.) between the maintenance supporting entities.

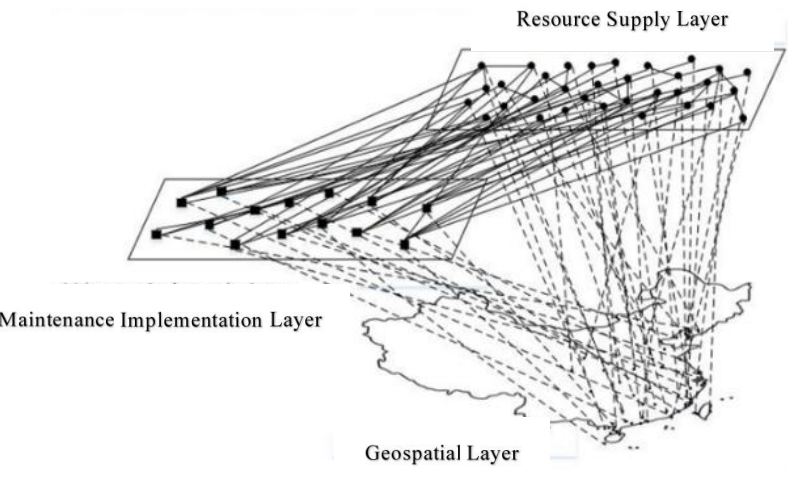

Fig.1 The maintenance support network model based on geospatial layer

The maintenance support network diagram is represented by $\mathrm{G}=(\mathrm{V}, \mathrm{E})$, where $\mathbf{V}=\left\{\boldsymbol{v}_{1}, \boldsymbol{v}_{2}, \ldots, \boldsymbol{v}_{\boldsymbol{n}}\right\}$ is a finite set of elements; $\boldsymbol{v}_{1}, \boldsymbol{v}_{2}, \ldots, \boldsymbol{v}_{\boldsymbol{n}}$ represents the participating entity node in the maintenance support system; $\mathbf{E}=\left\{\boldsymbol{e}_{1}, \boldsymbol{e}_{2}, \ldots, \boldsymbol{e}_{\boldsymbol{m}}\right\} \quad$ is a finite edge set corresponding to the node pairs in set $\mathrm{V}$, representing the interaction relationship based on different attributes[6].

In the network, with the change of time series, the dynamic evolution process of network is formed because of the addition and exit of network nodes. As an operation and maintenance supply chain network, the evolution behaviour of its nodes still follows the behaviour pattern of the general network structure: add (remove) the nodes, add (remove) the edges, re-connect. To some extent, these forms of evolution may exist at the same time.

Add a new vertex to the supply chain network, that is, the introduction of new manufacturers in the supply chain system and add a number of edges that depart from this vertex. Add edge refers to a number of new connections between the original vertices in the supply chain network, that is, the formation of a new supply or demand relationship in the supply chain system. Reconnect refers to breaking the original edge and then add another new edges to the same vertex, which means the manufacturer is excluded from the supply chain system and then it will re-join into the supply chain system. It is shown in Tab.1.

Table 1 The evolution model of supply chain network

\begin{tabular}{|c|c|}
\hline Network representation & Evolutionary behavior and its significance \\
\hline Add the nodes & New fundraising for node enterprises. \\
\hline $\begin{array}{c}\text { Remove the nodes } \\
\text { Add/remove the edges \& } \\
\text { Re-connect. }\end{array}$ & Old enterprise nodes were eliminated because they could not meet the needs of the \\
network chain.
\end{tabular}


As a network of economic relations, supply chain network has obvious unequal time and preference for node arrival. In the process of supply chain network evolution, it is the source power of evolution for member enterprises with limited rationality to seek the optimization of their own enterprise profit. Therefore, the evolution of supply chain network shows a certain degree of concentration and randomness. The core vendors get most of the connections while other vendors get fewer connections because of the different status of nodes and the roles they assume in the supply chain. It is consistent with the actual supply chain system. For the existing manufacturers in system, the fluctuation of the manufacturer's state will also cause the departure of the original manufacturer, which is manifested as the break of the link and the detachment of the manufacturer in the supply chain network. When the manufacturer is out of the supply chain system, accordingly, the vendor node and its connection related to the node are deleted in the network.

It is assumed that the process of nodes joining and leaving supply chain system obeys the Poisson distribution. Therefore the rules for building maintenance network evolution are as follows [7]:

1) Given supply chain network system, it includes a number of $\boldsymbol{m}_{\mathbf{0}}$ enterprise nodes $(\mathrm{t}=0)$.

2) Growth: Per time step, there is a new node added to the network. It is connected with other existing $\mathbf{m}$ nodes. $\left(\mathbf{m}<\boldsymbol{m}_{\mathbf{0}}\right)$.

3) Connection priority: The probability that new node is connected to the existing node $\mathbf{i}$ is proportional to $\boldsymbol{k}_{\boldsymbol{i}}$. That is, the actual connection is affected by the node connection priority. $\prod_{i}=\frac{\boldsymbol{k}_{i}}{\sum_{j} \boldsymbol{k}_{j}}$.

\section{Collaborative Efficiency Evaluation of maintenance supply chain network}

Interaction between nodes is quote complex in the real marine maintenance support system. The traditional maintenance support focus on the local optimization of single node, however, the maintenance support system in complex network environment has a higher requirement on the information sharing and collaboration among every maintenance support unit. As a result, a moderate collaboration should be found in the premise that the reliability of maintenance could be ensured so that an optimizing strategy could be achieved for the operation and maintenance cost and system efficiency [8] [9].
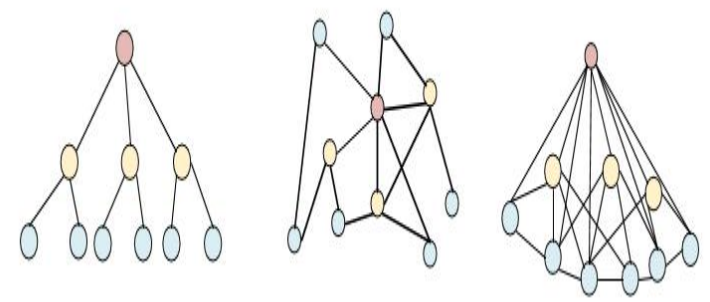

Fig.2 The maintenance support network synergy

In the traditional chained maintenance support system, the maintenance support entities lack collaborative relations. That is to say there is only superior and subordinate support relations among the entity nodes without the collaboration, information sharing and resource sharing. This kind of maintenance support system is low cost, small profit, and low efficiency. The opposite network has larger length of feature path and synchronized collaborative index, and low cost.

Moderate collaboration develops the sharing collaborative relations among limited information, resource and maintenance capability. The cost of the systems in this mode increases moderately, but the profit and efficiency increase significantly. The network characteristics in this mode has moderate length of feature path and synchronized collaborative index, and lower cost.

In excessive collaboration, all nodes in the maintenance support system tend to be in all connected mode based on the moderate collaboration. There is an interaction of information and resources between all maintenance support entities. The excessive collaboration maintenance network results in ultra-high costs without benefits improved accordingly. The network characteristics in this mode has lower length of feature path and maximum collaborative index, and higher cost.

For the maintenance support network, the synergy efficiency is mainly embodied in the following 3 aspects: the difficulty of the synergistic realization, the size of the synergistic cost, the advantages and disadvantages of the synergy effect [10]. Therefore, according to the definition of complex network, we should choose the appropriate performance index to establish the evaluation model of maintenance support network and calculate the overall topological evolution characteristics statistically. Then construct the best cooperative maintenance support network model. The evolutionary algorithm flow is shown in the figure 3: 


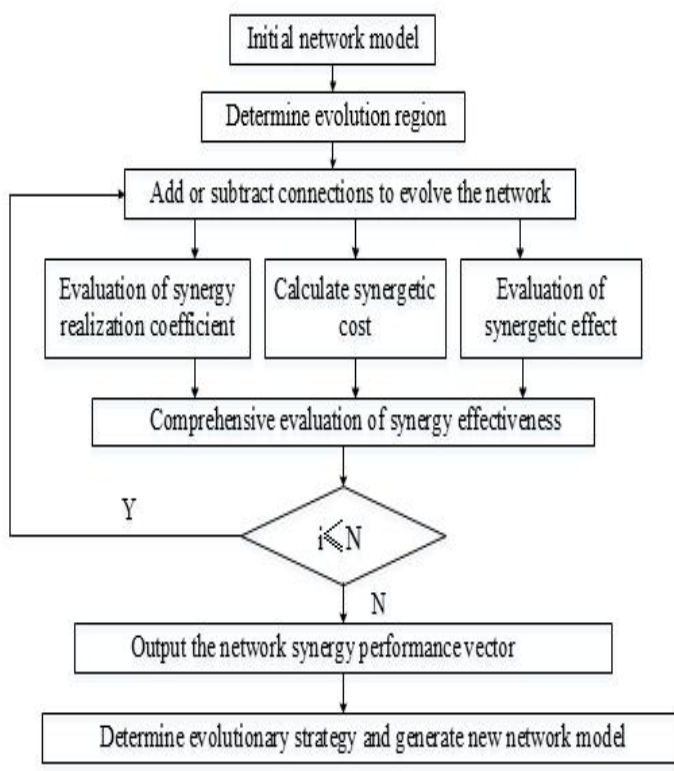

Fig.3 The maintenance support network evolutionary algorithm

1) On the basis of the initial network model, the network evolution area is determined.

2) In the evolutionary region, the evolution strategy is implemented in turn 1) to obtain the new network model and to calculate the collaborative efficiency under the new model. Then it generates the network synergy performance vector.

3) The region corresponding to the optimal value in network synergy performance vector is the optimal strategy of network collaborative evolution.

4) Iteration of the above process across the network, then global evolution strategy is determined and the collaborative performance curve in the process of optimal network evolution is calculated. Finally the support network with the greatest synergy efficiency is designed under the optimal collaborative evolution strategy.

\section{Conclusion}

Maintenance support is a solid foundation for the safe and efficient operation. The research of maintenance support network model based on supply chain perspective shows the core maintenance base and spare parts suppliers have obvious connection priority in the network structure and supply relationship. The complex characteristics of organization, self-adaptation and dynamic evolution affect the operation efficiency of the whole network to a certain extent. Also more research on supply network structure evolution and supply chain cooperative game based on collaboration will be developed.

\section{References}

1. Barabási A L, Albert R, Jeong H. Evolution of the social network of scientific collaborations [J]. Physical A,1999: 272-273.

2. Dekker R. Applications of maintenance optimization models: a review and analysis [J]. Reliability Engineering and System Safety, 1996, 51(3): $229-240$.

3. Xiaohu Yin. Research on the Dynamical Analysis Method for Equipment Maintenance Systems [D]. Hunan: Graduate School of National University of Defence Technology, 2008.

4. Tao Zhang. Modelling and Analysis of Maintenance Support Capability Assessment of Equipment in the Usage Phase. [D]. Hunan: Graduate School of National University of Defence Technology, 2004.

5. Sui Rubin, Zhong Guijuan. Research on networks of parts relation for product family based on complex networks [J].Commercial Research, 2009(1) :5053.

6. WANG Xiaofan, LI Xiang, CHEN Guanrong. Complex network theory and its application [M].Beijing: Tsinghua University Press, 2006.

7. Kwon O, Im G P, Lee K C. MACE-SCM: A multi-agent and case-based reasoning collaboration mechanism for supply chain management under supply and demand uncertainties [J].Expert Systems with Applications, 2007, 33(3):690 -705.

8. GUO Lei , XU Xiao-ming. Complex networks[M].Shanghai: Shanghai Science Education Press, 2006.

9. Xu Yuguo. Research on key Technologies for Equipment Autonomic Logistics [D]. Hunan: Graduate School of National University of Defense Technology, 2008.

10. Hanaki N, Peterhansl A, Dodds P S, et al. Cooperation in evolving social networks [J]. Management Science, 2007, 53(7):1036 - 1050. 